\title{
Wideband Air-Ground Channel Model for a Regional Airport Environment
}

\author{
Nicolas Schneckenburger, Member, IEEE, Thomas Jost, Member, IEEE, Michael Walter, Member, IEEE, \\ Giovanni del Galdo, Member, IEEE, David W. Matolak, Member, IEEE, and Uwe-Carsten Fiebig, Member, IEEE
}

\begin{abstract}
In the air-ground propagation channel, multipath propagation severely affects the performance of communication, navigation, and surveillance systems used in civil aviation. To verify the performance of aeronautical wireless transmission systems independently from expensive flight trials a channel model for computer simulations is required. Based on an exhaustive propagation measurement campaign we propose a geometry-based stochastic channel model for the air-ground channel in a regional airport environment in the L-band. The proposed channel model allows to correctly reproduce the non-stationarity of the channel impulse response. Thus the channel model is suitable not only to assess the performance of communication systems, but also for ranging, i.e., for navigation systems. We validate the channel model using data from both channel sounding and as well as flight measurements performed in a navigation context.
\end{abstract}

\section{INTRODUCTION}

The way we use and manage the civil airspace is currently undergoing a major modernization process. A continued increase of the number of airspace users, e.g., air transportation and unmanned aerial vehicles (UAVs), mandates an improved operational efficiency. The change in use and increasing user numbers also mandate changes in the way the airspace is managed. In that context, the communication, navigation, and surveillance (CNS) infrastructure is a key factor, as it has to support more aircraft and thus has to provide better navigation accuracy and higher communication capacity compared to the state of the art. Therefore, the CNS infrastructure is currently undergoing a major innovation process.

On the communication side, analog transceivers developed in the late ' 30 s working in the very high frequency (VHF) band for voice-only links between pilot and air traffic controller will be replaced by L-band Digital Aeronautical Communication System (LDACS) [1].

On the navigation side, the largest part of the modernization is the transitioning from analog ground-based systems towards digital global navigation satellite systems (GNSS), e.g., Global Positioning System (GPS) or Galileo. Nevertheless, terrestrial based radionavigation will still play an important role in alternative positioning, navigation, and timing (APNT) systems within the future navigation infrastructure [2].

N. Schneckenburger, T. Jost, M. Walter, and U.-C. Fiebig are with the Institute of Communications and Navigation of the German Aerospace Center (DLR), Oberpfaffenhofen, Wessling, Germany. G. del Galdo is with the Institute for Information Technology, Ilmenau University of Technology, Ilmenau, Germany and the Fraunhofer Institute for Integrated Circuits IIS, Erlangen, Germany. D. Matolak is with the University of South Carolina, Columbia, SC. Copyright (c) 2015 IEEE. Personal use of this material is permitted. However, permission to use this material for any other purposes must be obtained from the IEEE by sending a request to pubs-permissions@ieee.org
Major factors limiting the performance of terrestrial CNS systems are the propagation conditions for radio waves traveling between the ground station and the aircraft, i.e., the airground (AG) channel. In fact, CNS radio signals traveling from the ground station to the aircraft may be altered or degraded, e.g., by multipath components (MPCs) or shadowing. The socalled AG channel describes how the transmit signal is affected by the propagation medium.

A general, comprehensive, and accurate model of the AG channel is mandatory to test and optimize modernized legacy systems or new systems. Existing AG channel models based on measurement data apply a non-geometric based statistical approach for most propagation effects [3]-[6] . However by using a purely statistical approach it is difficult to assess the performance of modern tracking based range estimators like [7], [8] that employ physical ray based propagation models inside the estimation. Therefore a geometric-based channel model is better suited.

A geometry-based stochastic channel model (GBSCM) incorporates a geometrical relation for individual MPCs represented by single point allowing for a realistic simulation of MPCs in non-stationary scenarios [9], [10]. A GBSCM models some properties of the MPC such as delay according to a simplified ray-tracing method. Examples for GBSCMs are given in [11]-[15].

In this paper, we propose a GBSCM for the AG channel suitable for communication and navigation applications transmitting in the L-band. The parameterization of the GBSCM is based on airborne channel measurements conducted in 2013 [16]. To assess the quality the channel model, it is successfully validated against measurement data from the channel measurements described in [16]. Furthermore, we present the performance of LDACS as obtained by measurements and via simulation with the proposed channel model [17]. We show that the proposed AG channel model is of high value for the computer-based performance analysis of CNS systems.

The paper is organized as follows: In Sec. II we give a brief summary of the GBSCM architecture. Sec. III shows how the parameterization of the AG channel model for a regional airport environment is derived from measurement data. The parameterization is validated in Sec. IV The conclusion and outlook are given in Sec. V.

\section{Channel Model Architecture}

The channel model uses a geometry-based stochastic approach: each propagation effect is represented by a geometrical 


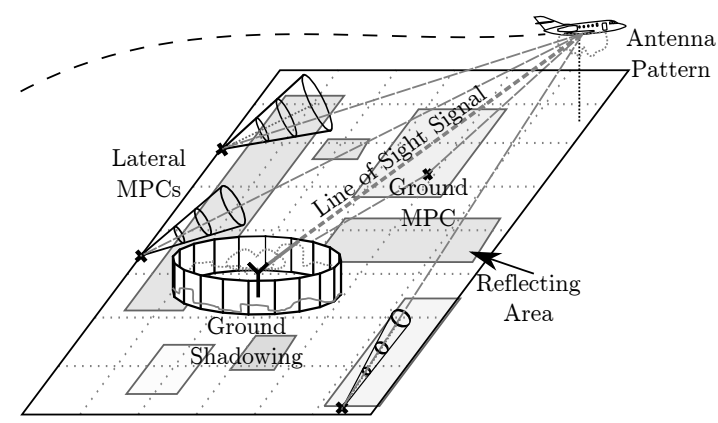

Fig. 1. Proposed GBSCM architecture for the AG channel.

model element. We draw the model element's properties, e.g., location of a reflector, from statistical distributions. These distributions are derived from measurement data [16]. We call the entirety of the statistical distributions describing all properties of a model element as a parameterization. A parameterization is thus environment specific: different parameterizations allow us to use the same GBSCM architecture to model different environments. In this paper we present a parameterization for a regional airport environment.

We talk of a realization of the channel model if we draw values for all model elements, e.g., positions of reflectors, from the corresponding distributions. After this step the properties of all model elements remain fixed - apart from noise, the channel is deterministic: the channel impulse response (CIR) is solely a function of the aircraft position and attitude.

Fig. 1 shows the proposed architecture of the channel model [18]. The general idea is to model the propagation channel as a sum of plane waves. The waves present different propagation effects, such as line-of-sight $(\mathrm{LoS})$ propagation, ground multipath propagation, and lateral multipath propagation, described in Sec. III-A through Sec. III-C.

Propagation paths are weighted by an antenna radiation pattern at the aircraft and an antenna radiation pattern at the ground station described in Sec. III-D. Additionally, the LoS path may be blocked by the effect of ground shadowing as outlined in Sec. III-E. The colored noise component described in Sec. III-F models diffuse MPCs.

The simulation chain of the channel model shown in Fig. 2 can be divided into three steps.

To initialize the channel model a parameterization for an environment is required. This parameterization includes all required statistical distributions describing model element properties and other information, e.g., information about the antennas. From the distributions describing the model element properties a realization of the channel model is generated: we draw the properties of ground MPC, the lateral MPCs, and diffuse MPCs, e.g., location of ground reflecting areas or the power associated with each reflector. After the initialization the channel model is fully defined: the channel characteristics are - apart from noise - only a function of the aircraft position and attitude.

In the next step the CIRs is generated. To this end, we first calculate the aircraft position and attitude for every time instance for which the channel is to be simulated. The positions and attitudes are interpolated based on the aircraft

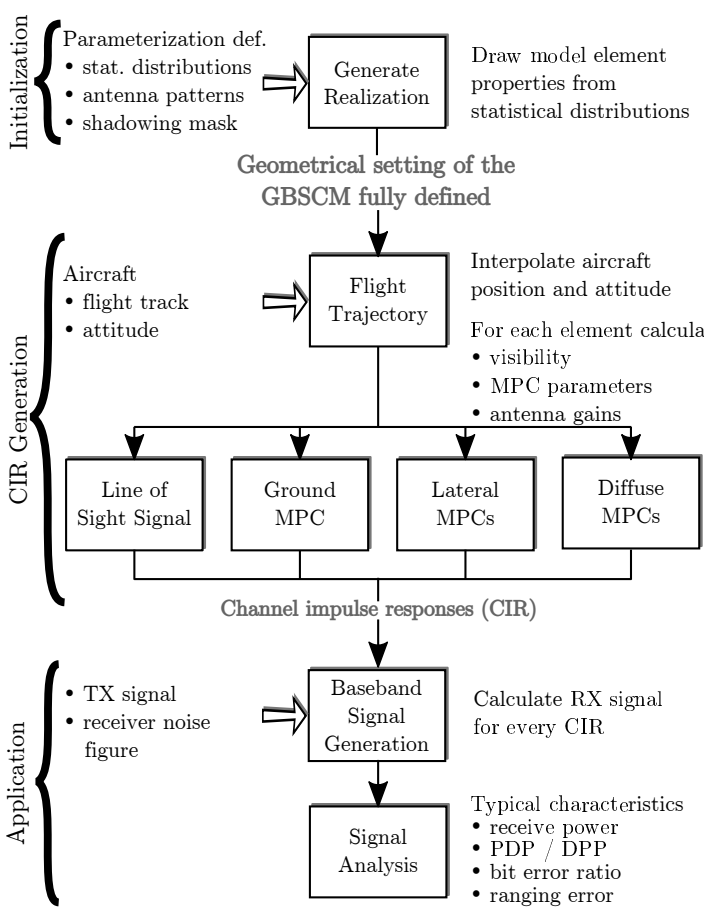

Fig. 2. The three steps of the simulation chain of the channel model.

trajectory defined by the user. For every time instance and given aircraft position and attitude, the geometrical nature of the channel model allows the calculation of the following MPC parameters: delay, Doppler frequency, and complex weight, and the gains of the involved antennas.

In the application step we convolve the CIRs with the desired transmit signal. This transmit signal may be a channel sounding signal to reproduce the channel sounding experiments, or the signal of a terrestrial CNS system. The generated receive signal can then be analyzed.

\section{Computational Complexity}

To provide a very rough indication on the computational complexity we run the channel model implemented in Mathworks Matlab ${ }^{\circledR} 2016 \mathrm{a}$ for a $1 \mathrm{~h}$ flight trajectory with a temporal resolution of $1 \mathrm{~Hz}$, i.e., a CIR is calculated 3600 times. The simulation is run on a laptop computer from the year 2014 (Intel $^{\circledR}$ Core $^{\mathrm{TM}} \mathrm{i} 7-4600,8$ GB RAM).

During the initialization step the most computationally complex task is to distribute the reflecting areas; this task takes up about $95 \%$ of the $90 \mathrm{~s}$ required for initialization step.

The generation of the 3600 CIRs takes roughly 8 min with almost the entire time required for the processing of lateral MPCs: for every time instance, depending on the angle the aircraft is seen under from a reflector, it has to be evaluated if a signal from that reflector is received.

The time required for the application step obviously strongly depends on the characteristics of the analyzed signal, e.g., the signal bandwidth. To provide a rough idea on the computational complexity of the application step we employ the LDACS signal. For every CIR we generate the receive signal assuming 50 LDACS symbols are transmitted. Using the generated receive signal we perform a maximum likelihood 
(ML) estimation of delay, Doppler frequency and complex amplitude. The application, i.e., the generation and analysis of the baseband signal lasts for roughly 2 min.

\section{Derivation OF THE PARAMETERIZATION}

In the following we derive a regional airport parameterization for the channel model. To this end, we go through all model elements defined in Sec. II and derive the associated distributions. We base each of the distributions on results from the channel sounding experiments described in [16].

Note that all distributions mentioned in the following are normalized probability density functions (PDFs), i.e., they integrate to ' 1 '. We assume the PDFs for the different properties to be independent, the joint PDF factorizes into the PDFs of the individual properties: as an example assume a ground reflecting area of logarithmic aspect ratio $\log \frac{l_{\mathrm{G}, 1}}{l_{\mathrm{G}, 2}}$, size $l_{\mathrm{G}, 1} \times l_{\mathrm{G}, 2}$, roughness $\sigma_{\mathrm{G}, \mathrm{r}}$, material $\mathrm{m}$. The joint $\mathrm{PDF}$ is then

$$
\begin{aligned}
& p\left(\log \frac{l_{\mathrm{G}, 1}}{l_{\mathrm{G}, 2}}, l_{\mathrm{G}, 1} \times l_{\mathrm{G}, 2}, \sigma_{\mathrm{G}, \mathrm{r}}, \mathfrak{m}\right)= \\
& \quad p\left(\log \frac{l_{\mathrm{G}, 1}}{l_{\mathrm{G}, 2}}\right) p\left(l_{\mathrm{G}, 1} \times l_{\mathrm{G}, 2}\right) p\left(\sigma_{\mathrm{G}, \mathrm{r}}\right) p(\mathfrak{m}) .
\end{aligned}
$$

Therefore, we can draw all properties separately from another.

Note also that for better illustration in plots angles are expressed in degrees. Nevertheless the parameter values for the statistical distributions assume angles to be expressed in radians.

For a better overview of the parameters and statistical distributions used in the channel model an overview is provided in Tab. I.

\section{A. Line-of-sight Signal}

We propose modeling the LoS signal power to follow free space path loss (FSPL). The phase of the LoS signal depends on the propagation path length and the signal wavelength. Additionally, if complex antenna radiation patterns are available, the LoS signal phase is a function of both angle of departure and angle of arrival.

If the absolute precise length of the LoS path is of interest, as it may be for the analysis of navigation systems, we have to consider the bending of electromagnetic waves due to the troposphere [19]. We propose modeling effects due to the troposphere using an established method from literature [20]. The results presented in [16] show that the effects of the troposphere can be well predicted.

Note that in the channel model the effect of the troposphere may be modeled not only for the LoS path, but also for all other MPCs .

\section{B. Ground Multipath Component}

We propose modeling ground multipath propagation by characterization of reflecting areas on the ground. A ground MPC is received if the ground reflection point lies in such a reflecting area. We calculate the location of the ground reflection point as the specular reflection point on the ground

\begin{tabular}{|c|c|}
\hline \\
\hline & \\
\hline \\
\hline \\
\hline \\
\hline \multirow{2}{*}{\multicolumn{2}{|c|}{$\begin{array}{l}\text { Ground MPC } \\
\zeta_{\mathrm{G}, \mathrm{AR}} \\
\zeta_{\mathrm{G}, \mathrm{r}} \\
\zeta_{\mathrm{G}, s} \\
l_{\mathrm{G}, 1}, l_{\mathrm{G}, 2} \\
\mathfrak{m}\end{array}$}} \\
\hline & \\
\hline \multicolumn{2}{|l|}{ Lateral MPCs } \\
\hline$\alpha, \boldsymbol{\alpha}$ & Complex weight(s) of MPC(s) \\
\hline \multirow{2}{*}{$\gamma_{\mathrm{ar}}$} & Pole of AR process \\
\hline & Angle the aircraft is seen from the reflector \\
\hline \multirow{2}{*}{$\begin{array}{l}\zeta_{N_{\text {ref }}} \\
\zeta_{\theta_{\mathrm{d}}, 1}, \zeta_{\theta_{\mathrm{d}}, \mathrm{u}}\end{array}$} & Number of reflectors \\
\hline & $\begin{array}{l}\text { Elevation opening direction: upper and lower limit } \\
\text { of uniform distr. }\end{array}$ \\
\hline$\zeta_{\phi_{\mathrm{rd}}, \mathrm{b}}$ & $\begin{array}{l}\text { Azimuth opening dir:: scale param. of Laplacian } \\
\text { distr. }\end{array}$ \\
\hline$\zeta_{\omega, \mu}, \zeta_{\omega, \sigma}$ & $\begin{array}{l}\text { Opening width: mean and std. dev. of log-normal } \\
\text { distr. }\end{array}$ \\
\hline$\zeta_{\bar{\alpha}_{\mathrm{A}}, 1}, \zeta_{\bar{\alpha}_{\mathrm{A}}, 2}, \zeta_{\bar{\alpha}_{\mathrm{A}}, 3}$ & $\begin{array}{l}\text { Weight - average: coefficients of distance dep. } \\
\text { mean of average amplitude }\end{array}$ \\
\hline$\zeta_{\alpha_{\mathrm{An}}, \mu}, \zeta_{\alpha_{\mathrm{An}}, \sigma}$ & $\begin{array}{l}\text { Weight - average: mean and std. dev. of log-normal } \\
\text { distr. }\end{array}$ \\
\hline$\zeta_{\alpha_{\mathrm{V}, \varphi}, A}, \zeta_{\alpha_{\mathrm{V}, \varphi}, B}$ & $\begin{array}{l}\text { Weight - variation: scale and shape of Weibull } \\
\text { distr. describing AR-pole }\end{array}$ \\
\hline \multirow{4}{*}{$\begin{array}{l}\zeta_{\alpha_{\mathrm{V}, K}, \mu}, \zeta_{\alpha_{\mathrm{V}, K}, \sigma} \\
\zeta_{\alpha_{\mathrm{W}}, 1}, \zeta_{\alpha_{\mathrm{W}}, 2} \\
\zeta_{r, w 1}, \zeta_{r, w 2}, \zeta_{r, w 3} \\
\zeta_{r, 1, \sigma}\end{array}$} & $\begin{array}{l}\text { Weight - variation: mean and std. dev. of log- } \\
\text { normal distr. describing K-factor }\end{array}$ \\
\hline & Weight - window: coefficients of window function \\
\hline & Position: weights of different reflectors classes \\
\hline & $\begin{array}{l}\text { Position - close reflectors: scale parameter of half- } \\
\text { normal distr. }\end{array}$ \\
\hline$\zeta_{r, 2, N}$ & $\begin{array}{l}\text { Position - cluster reflectors: number of components } \\
\text { in Gaussian mixture }\end{array}$ \\
\hline$\zeta_{r, 2, \mu}, \zeta_{r, 2, \sigma}$ & $\begin{array}{l}\text { Position - cluster reflectors: mean and std. dev. of } \\
\text { components in Gaussian mixture }\end{array}$ \\
\hline$\zeta_{r, 3,1}, \zeta_{r, 3, \mathrm{u}}$ & $\begin{array}{l}\text { Position - isolated reflectors: lower and upper } \\
\text { bound of uniform distr. }\end{array}$ \\
\hline$\theta$ & Elevation angle (as seen from reflector) \\
\hline$\phi$ & Azimuth angle (as seen from reflector) \\
\hline & Angle defining chord of a circle \\
\hline \multirow{2}{*}{ 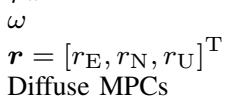 } & Opening width (of reflector) \\
\hline & Reflector location in ENU \\
\hline \multirow{2}{*}{$\begin{array}{l}\zeta_{\mathrm{CN}, \mathrm{y}}, \zeta_{\mathrm{CN}, \mathrm{s}} \\
\zeta_{\mathrm{CN}, \sigma}\end{array}$} & $y$-intersection and slope of exponential function \\
\hline & $\begin{array}{l}\text { Std. dev. of normal distribution describing scatterer } \\
\text { location }\end{array}$ \\
\hline $\begin{array}{l}\boldsymbol{r}_{\mathrm{CN}}=\left[d_{\mathrm{CN}, \mathrm{E}}, . .\right. \\
\left.d_{\mathrm{CN}, \mathrm{N}}\right]^{\mathrm{T}}\end{array}$ & Location of scatterer \\
\hline \multirow{8}{*}{$\begin{array}{l}\text { Distributions } \\
\mathcal{E} \\
\log \mathcal{N} \\
\mathcal{L} \\
\mathcal{N} \\
|\mathcal{N}| \\
\mathcal{U} \\
\mathcal{W}\end{array}$} & Parameters are given in brackets $(\cdot)$ \\
\hline & Exponential distribution (mean) \\
\hline & Log-normal distribution (mean and std. dev.) \\
\hline & Laplace distribution (scale) \\
\hline & Normal distribution (mean and std. dev.) \\
\hline & Half-normal distribution (scale) \\
\hline & Uniform distribution (lower and upper limit) \\
\hline & Weibull distribution (scale and shape) \\
\hline
\end{tabular}
surface [19]. The location of the ground reflection point
TABLE I. List of parameters and statistical distributions used in the channel model.

depends on both ground antenna height and aircraft position. We assume horizontally aligned reflecting areas. Thus, once the reflecting areas are defined, the interaction of the ground MPC with the LoS signal, and the resulting attenuation or amplification of the latter, follows directly from the underlying geometry.

We define the coverage ratio $r_{\mathrm{G}, \mathrm{cov}}$ as the ratio between the sum of all reflecting area sizes and the overall area size around the ground station. For the regional airport environment, based on the measured data, we approximate that $r_{\mathrm{G}, \mathrm{cov}}=50 \%$ of the ground station surrounding is covered by reflecting areas. For normal flight situations choosing an overall area size of 
$5 \mathrm{~km} \times 5 \mathrm{~km}$ is usually adequate.

We characterize the reflecting areas by four different properties. We derive the distribution of the area properties based on ground surface information of the environment to be modeled. In our case we use land usage data, satellite imagery, and a digital elevation model (DEM) of the ground station surroundings.

1) Shape: The reflecting areas (such as a runway, a parking area, a taxiing area) are of different shape. For simplicity we model all reflecting areas as rectangles of size $l_{\mathrm{G}, 1} \times l_{\mathrm{G}, 2}$. We use a normal distribution with standard deviation $\zeta_{\mathrm{G}, \mathrm{AR}}$ to describe the logarithmic aspect ratio of each rectangle

$$
p\left(\log \frac{l_{\mathrm{G}, 1}}{l_{\mathrm{G}, 2}}\right) \sim \mathcal{N}\left(0, \zeta_{\mathrm{G}, \mathrm{AR}}=0.1\right) .
$$

Thus, reflecting areas are all of rectangular shape each with a different aspect ratio. Using a statistical distribution to model the logarithm of the aspect ratio - rather than the aspect ratio itself - guarantees two important properties: first, the lengths of both sides of the rectangle are equally distributed, i.e., $p\left(l_{\mathrm{G}, 1}\right)=p\left(l_{\mathrm{G}, 2}\right)$. second, the resulting shapes of the ground reflecting areas are representative for the regional airport environment.

2) Size: The airport environment we are modeling consists predominantly of large open areas of either grassy or concrete surface. Thus, also the reflecting areas in the channel model are large: We model the size $l_{\mathrm{G}, 1} \times l_{\mathrm{G}, 2}$ as an exponential distribution with mean $\zeta_{\mathrm{G}, s}$

$$
p\left(l_{\mathrm{G}, 1} l_{\mathrm{G}, 2}\right) \sim \mathcal{E}\left(\zeta_{\mathrm{G}, s}=0.001 \mathrm{~km}^{2}\right) .
$$

Therefore, the average size of a reflecting area, i.e., the expectation value and mean of $p\left(l_{\mathrm{G}, 1} l_{\mathrm{G}, 2}\right)$, would - in the case of an aspect ratio of ' 1 ' - correspond to a $30 \mathrm{~m} \times 30 \mathrm{~m}$ square. Note however that all ground reflecting areas are of rectangular shape with one longer and one shorter side.

3) Material: The ground station environment is usually composed of a mix of different types of surfaces. Their electromagnetic properties such as conductivity and permittivity have been extensively investigated in the past [21], [22] and are modeled by statistical distributions. The ground station environment consists mainly of grassy areas, fields, forests, and concrete. Therefore, we assume that the reflecting areas consists of equal parts of medium dry ground, dry ground, and concrete as defined in [22].

With the integers 1,2 , and 3 indexing the three types of ground surfaces - medium dry ground, dry ground, and concrete - the PDF $p(\mathfrak{m})$ describing the distribution of material $\mathfrak{m}$ of a reflecting area can be expressed as

$$
p(\mathfrak{m})=\left\{\begin{array}{ll}
\frac{1}{3} & \text { for } \mathfrak{m} \in\{1,2,3\} \\
0 & \text { else }
\end{array} .\right.
$$

4) Roughness: The roughness of an area has an influence on how much power is scattered in all directions rather than reflected in the direction defined by the law of reflection [19]. We describe the roughness by the standard deviation $\sigma_{\mathrm{G}, \mathrm{r}}$ of the surface height about the local surface mean. Given $\sigma_{\mathrm{G}, \mathrm{r}}$ the reflected power can be approximated [21]. We model the

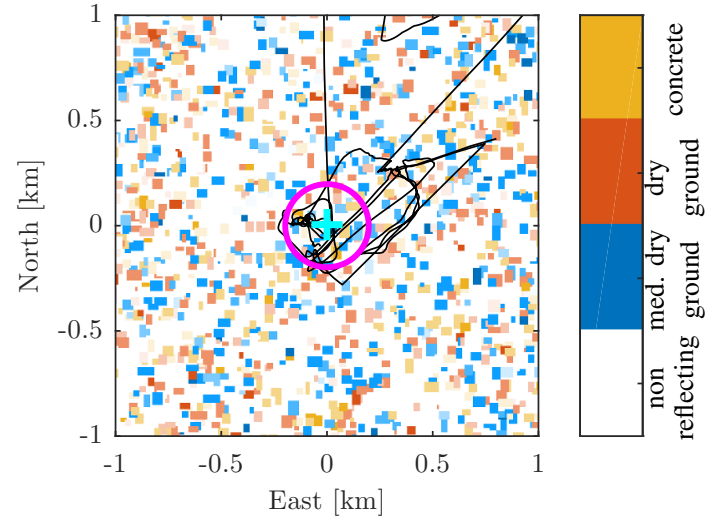

Fig. 3. Example for a ground reflecting area realization in the channel model.

standard deviation of the surface height using an exponential distribution with mean $\zeta_{\mathrm{G}, \mathrm{r}}$

$$
p\left(\sigma_{\mathrm{G}, \mathrm{r}}\right) \sim \mathcal{E}\left(\zeta_{\mathrm{G}, \mathrm{r}}=0.1 \mathrm{~m}\right) .
$$

The mean of 0.1 has been found to provide a good approximation in similar scenarios [23].

Fig. 3 shows one realization of the ground reflecting areas for the above property distributions [24]. The different colors in Fig. 3 represent different ground surface materials. The lightness of the areas indicates the roughness, i.e., the standard deviation $\sigma_{\mathrm{G}, \mathrm{r}}$ of the surface height about the local surface mean: with increasing roughness surfaces become lighter. The black line indicates the track of the ground reflection point for the conducted channel sounding flights. The magenta line shows the ground reflection point track for a hypothetical flying a circle around the ground station at $5 \mathrm{~km}$ above ground level (AGL) at a link distance of $50 \mathrm{~km}$, i.e., the distance between ground station and aircraft.

\section{Lateral Multipath Component}

In this section, we derive the statistical distributions describing the lateral MPCs. We propose representing lateral components by point reflectors. The visibility of a reflector is defined by a cone shaped opening [15]. Thus, the number of visible lateral MPCs and their MPC parameters is a function of the aircraft position. For the derivation of the statistical distributions we use information from approximately 130.000 individual reflectors. The reflectors are localized using the Bayesian method presented in [25].

Throughout this section we use the following notation:

- The estimated position of reflector $k$ in an east-northup (ENU) coordinate system is denoted by the vector $\boldsymbol{r}_{k}=\left[r_{k, \mathrm{E}}, r_{k, \mathrm{~N}}, r_{k, \mathrm{U}}\right]^{\mathrm{T}} \in \mathbb{R}^{3 \times 1}$.

- $M_{k} \in \mathbb{N}$ denotes the number of channel sounding blocks in which the MPC caused by reflector $k$ is detected. Thus, $M_{k}$ is equal to the duration the MPC is received divided by the block duration $T_{\text {sym }}$.

- The vector $\hat{\boldsymbol{\alpha}}_{k}=\left[\hat{\alpha}_{k, 1}, . ., \hat{\alpha}_{k, M_{k}}\right]^{\mathrm{T}} \in \mathbb{R}^{M_{k} \times 1}$ denotes the estimated complex weights associated with reflector $k$.

- The vectors $\hat{\phi}_{k}=\left[\hat{\phi}_{k, 1}, . ., \hat{\phi}_{k, M_{k}}\right]^{\mathrm{T}} \in \mathbb{R}^{M_{k} \times 1}$ and $\hat{\boldsymbol{\theta}}_{k}=\left[\hat{\theta}_{k, 1}, . ., \hat{\theta}_{k, M_{k}}\right]^{\mathrm{T}} \in \mathbb{R}^{M_{k} \times 1}$ denote the azimuth and 


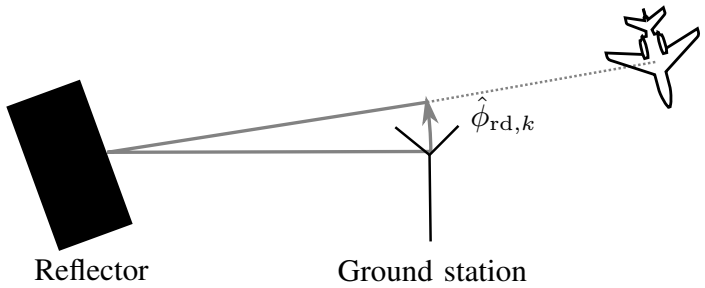

Fig. 4. Visualization of the relative opening direction $\hat{\phi}_{\mathrm{rd}, k}$.

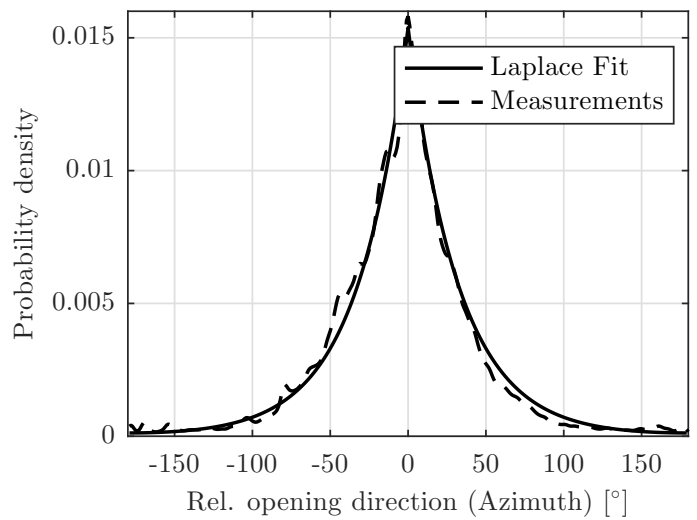

Fig. 5. Distribution of the relative azimuthal opening angle $\phi_{\mathrm{rd}, k}$.

elevation the aircraft is seen from the reflector $k$ while an MPC from the reflector $k$ is received.

- The elevation the aircraft is seen from the ground station while an MPC from reflector $k$ is received is denoted as $\hat{\theta}_{\mathrm{GS}, k}=\left[\hat{\theta}_{\mathrm{GS}, k, 1}, . ., \hat{\theta}_{\mathrm{GS}, k, M_{k}}\right]^{\mathrm{T}} \in \mathbb{R}^{M_{k} \times 1}$.

1) Number of Reflectors: The number of reflectors $\zeta_{N_{\text {ref }}}$ we distribute in the channel model is a key property of the channel model parameterization: $\zeta_{N_{\text {ref }}}$ defines how many MPCs are received. We adjust the $\zeta_{N_{\text {ref }}}$ such that the average number of reflectors detected during the measurements matches the number of reflectors seen during channel model simulations. An analysis of different flight segments show that $\zeta_{N_{\mathrm{ref}}}=$ $3.8 \cdot 10^{4}$ provides a good match.

2) Opening Direction: The opening direction is the axis of symmetry of the cone used to model a reflector. We divide the opening direction in an azimuthal and elevational direction, $\hat{\phi}_{\mathrm{d}, k}$ and $\hat{\theta}_{\mathrm{d}, k}$, respectively.

a) Azimuth: For the purpose of modeling we calculate the azimuthal opening direction relative to the direction the ground station is seen under as visualized in Fig. 4 as

$$
\phi_{\mathrm{rd}, k}=\hat{\phi}_{\mathrm{d}, k}-\tan _{2}^{-1}\left(-r_{k, \mathrm{~N}},-r_{k, \mathrm{E}}\right) .
$$

Thus, $\hat{\phi}_{\mathrm{rd}, k}=0$ means that aircraft, ground station, and reflector lie on a line while an MPC is received from the reflector.

In Fig. 5 we show the distribution of the relative azimuthal opening direction $p\left(\hat{\phi}_{\mathrm{rd}}\right)$. The estimated distribution of $p\left(\hat{\phi}_{\mathrm{rd}}\right)$ is symmetrical around $0^{\circ}$, i.e., most MPCs are reflected back towards the ground station. This effect, in literature referred to as backscattering, was also observed in previous measurements and simulations [26]. Simple ray-

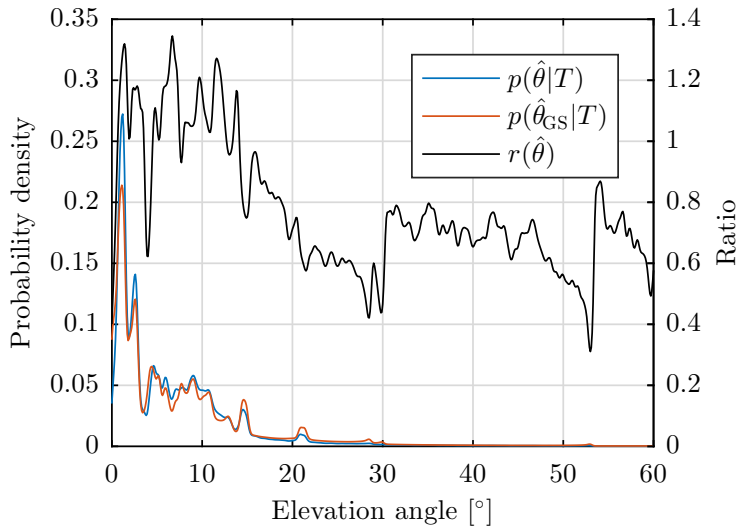

Fig. 6. Distribution of the elevation angle the aircraft is seen from both ground station and reflector positions.

tracing simulations indicate that the narrow distribution shown in Fig. 5 are most likely caused by the underlying geometry.

The distribution of $p\left(\phi_{\mathrm{rd}}\right)$ is modeled using a Laplacian distribution $\mathcal{L}$ centered at $0^{\circ}$, i.e., two exponential distributions mirrored around the $y$-axis,

$$
p\left(\phi_{\mathrm{rd}}\right) \sim \mathcal{E}\left(0, \zeta_{\phi_{\mathrm{rd}}, \mathrm{b}}=0.57\right) .
$$

Note that due to the $2 \pi$ periodicity of the angle $\phi_{\mathrm{rd}}$, we wrap the tails of the distribution around in order to make the distribution cyclic.

b) Elevation: The derivation of the distribution for the elevational opening direction $p\left(\theta_{\mathrm{d}}\right)$ is more cumbersome as for the azimuthal direction $p\left(\phi_{\mathrm{d}}\right)$. The complication is caused by the fact that during the measurements the aircraft is only seen under very specific elevation angles from the ground station: the estimated distribution of elevation angles is strongly dependent on the measured flight trajectory $T$. In contrast, the azimuth angles $\phi$ the aircraft is seen from the ground station is approximately uniformly distributed from 0 to $2 \pi$.

Fig. 6 (left $y$-axis) shows two estimated distributions: first, the distribution of the elevation angle the aircraft is seen from the ground station $p\left(\hat{\theta}_{\mathrm{GS}} \mid T\right)$ during the entire flight. Second, the distribution of the elevation angle the aircraft is seen from all detected reflectors $p(\hat{\theta} \mid T)$. We use the notation of a conditional distribution to underline the dependence on the flight track $T$. For the calculation of the first distribution $p\left(\hat{\theta}_{\mathrm{GS}} \mid T\right)$ every location of the aircraft flight track is only considered once. In contrast, for the second distribution $p(\hat{\theta} \mid T)$ every location is weighted by the number of MPCs received while the aircraft is at said location.

We now aim to investigate the distribution of the elevational opening direction $p(\theta)$ independently from the flight track $T$. By applying Bayes law we write

$$
\frac{p(T \mid \hat{\theta}) p(\hat{\theta})}{p\left(T \mid \hat{\theta}_{\mathrm{GS}}\right) p\left(\hat{\theta}_{\mathrm{GS}}\right)}=\frac{p(\hat{\theta} \mid T) p(T)}{p\left(\hat{\theta}_{\mathrm{GS}} \mid T\right) p(T)} .
$$

Taking into consideration that the relative distance between ground station and reflector are usually small compared to the link distance, the elevation angles the aircraft is seen from the 


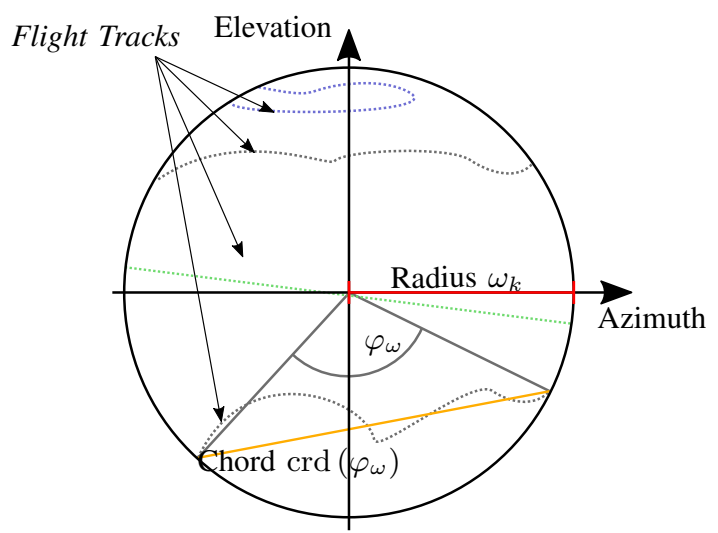

Fig. 7. Illustration of the opening width calculation: the figure illustrates different flight tracks as seen from the reflector position.

ground station, $\theta_{\mathrm{GS}}$, and reflector, $\theta$, are almost identical: it follows that

$$
p(T \mid \hat{\theta}) \approx p\left(T \mid \hat{\theta}_{\mathrm{GS}}\right) .
$$

Using the simplification from (9), we are able to rewrite (8) as

$$
r(\hat{\theta}):=\frac{p(\hat{\theta})}{p\left(\hat{\theta}_{\mathrm{GS}}\right)}=\frac{p(\hat{\theta} \mid T)}{p\left(\hat{\theta}_{\mathrm{GS}} \mid T\right)} .
$$

The ratio $r(\hat{\theta})$ is proportional to the number of reflectors seen by the aircraft under a certain elevation angle $\theta$ : if a dominant elevation angle $\theta^{\prime}$ exists under which the aircraft is seen from a large number of reflectors, there is a peak in the ratio $r(\theta)$ at that angle $\theta^{\prime}$. Consequently, reflectors with an elevational opening direction $\theta_{\mathrm{d}}^{\prime}$ are more likely than reflectors with other opening directions.

From Fig. 6 we observe that the ratio $r(\hat{\theta})$ is approximately constant $^{1}$. If the ratio $r(\theta)$ is constant, we can model the distribution of elevational opening directions $p\left(\theta_{\mathrm{d}}\right)$ as uniform distribution $\mathcal{U}$ between $\zeta_{\theta_{\mathrm{d}}, \mathrm{l}}=0$ and $\zeta_{\theta_{\mathrm{d}}, \mathrm{u}}=\frac{\pi}{2}$

$$
p\left(\theta_{\mathrm{d}}\right) \sim \mathcal{U}\left(\zeta_{\theta_{\mathrm{d}}, \mathrm{l}}=0, \zeta_{\theta_{\mathrm{d}}, \mathrm{u}}=\frac{\pi}{2}\right) .
$$

3) Opening Width: In this section, we describe how the distribution of the opening width $p(\omega)$ can be derived from the measured data. Seen from the reflector position the opening width defines a circle centered at $\left[\phi_{\mathrm{d}, k}, \theta_{\mathrm{d}, k}\right]^{\mathrm{T}}$ as visualized in Fig. 7. The radius of the circle equals the opening width $\omega_{k}$. If the aircraft traverses on a straight path through the circle and its center as shown by the green track in Fig. 7, the estimation of the opening width $\hat{\omega}_{k}$ is straightforward: it is the Euclidean distance between the first and last two-dimensional angle the aircraft is seen under

$$
\hat{\omega}_{k}=\sqrt{\left(\hat{\phi}_{k, 1}-\hat{\phi}_{k, M_{k}}\right)^{2}+\left(\hat{\theta}_{k, 1}-\hat{\theta}_{k, M_{k}}\right)^{2}} .
$$

\footnotetext{
${ }^{1}$ Although the true ratio $r(\theta)$ may not be perfectly constant, with the available measurement it is unfeasible to determine the exact shape of $r(\theta)$. Thus, the assumption of a constant ratio $r(\theta)$ is reasonable. For more exact determination ratio $r(\theta)$ more flight trials, especially with trajectories where the aircraft is seen at higher elevation angles from the ground station, would be required.
}

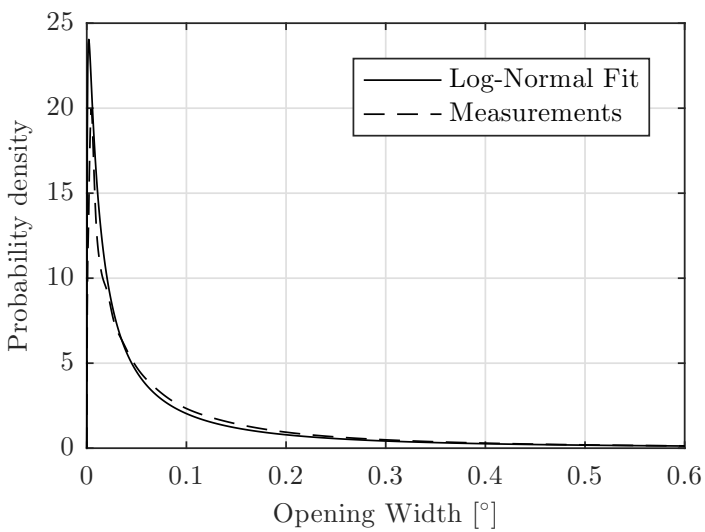

Fig. 8. Measured and modeled distributions of the opening width $\omega$.

In reality the aircraft will most likely traverse through the circle neither on a straight path nor through the center, e.g., blue or grey tracks. We will thus underestimate the opening width $\hat{\omega}_{k}$ in most cases if we use (12).

Nevertheless, we are able to estimate the opening width $\hat{\omega}_{k}$ based on the measured data as described below: as visualized in Fig. 7, each aircraft trajectory "through the circle" defines a chord $\operatorname{crd}\left(\varphi_{\omega}\right)$ (orange line). The length of the chord is given as

$$
\operatorname{crd}\left(\varphi_{\omega}\right)=2 \omega \sin \frac{\varphi_{\omega}}{2} .
$$

Assume that the angle $\varphi_{\omega}$ defining the chord length is uniformly distributed, i.e., $p\left(\varphi_{\omega}\right) \sim \mathcal{U}(0,2 \pi)$ : the average chord length can be calculated as

$$
\mathbb{E}\left\{\operatorname{crd}\left(\varphi_{\omega}\right)\right\}=\int_{0}^{2 \pi} \frac{1}{2 \pi} 2 \omega_{k} \sin \frac{\varphi_{\omega}}{2} \mathrm{~d} \varphi_{\omega}=\frac{4 \omega_{k}}{\pi} .
$$

Using (14) we are able to translate a chord length $\operatorname{crd}\left(\varphi_{\omega}\right)_{k}$ estimated from the measurements data into an opening width $\omega_{k}$.

The opening width $\omega_{k}$ is thus calculated as

$$
\begin{array}{r}
\hat{\omega}_{k}=\frac{\pi}{4} \max _{i, j} \sqrt{\left(\hat{\phi}_{k, i}-\hat{\phi}_{k, j}\right)^{2}+\left(\hat{\theta}_{k, i}-\hat{\theta}_{k, j}\right)^{2}} \\
\text { with } i, j=1, . ., M_{k} .
\end{array}
$$

The estimated distribution of the opening width $p(\hat{\omega})$ is shown in Fig. 8. We observe that most reflectors have a narrow opening width $\omega<0.1^{\circ}$. Nevertheless, due to a small number of reflectors with large opening widths $\omega$, the mean opening width is $0.7^{\circ}$. These reflectors have a significant influence on the channel, as due to their large opening width $\omega$, they cause MPC visible for a long duration. To model the distribution $p(\omega)$ we chose a log-normal distribution with mean $\zeta_{\omega, \mu}$ and standard deviation $\zeta_{\omega, \sigma}$

$$
p(\omega) \sim \log \mathcal{N}\left(\zeta_{\omega, \mu}=-7.15, \zeta_{\omega, \sigma}=1.77\right) .
$$

The $\log$-normal distribution $\log \mathcal{N}$ is chosen over other distributions as it provides also a good match for the tails of the estimated distribution $p(\hat{\omega})$, i.e., reflectors with large opening widths $\omega$. The drawback is a slight mismatch between the modeled and estimated distribution at smaller opening widths $\omega$ around $0.1^{\circ}$. 


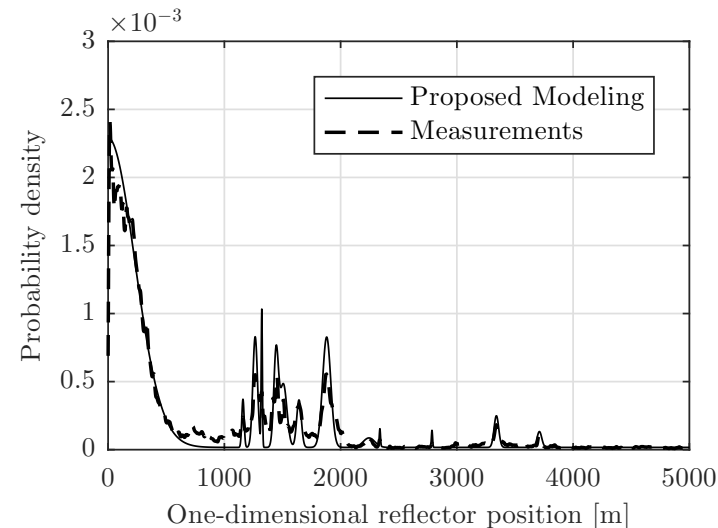

Fig. 9. Distribution of the one-dimensional reflector location, i.e., the distance between ground station and reflector.

4) Location: This section treats the modeling of the reflector location distribution $p(\boldsymbol{r})$. The goal is to model the distribution of positions $p(\boldsymbol{r})$ as simple as possible while maintaining a good match to the measured distribution $p(\hat{\boldsymbol{r}})$.

In the following we assume that all reflectors are located in the same plane, e.g., their altitude relative to the ground station antenna is constant. Furthermore, we model the originally then two-dimensional location using by an one-dimensional distribution $p(r)$, i.e., the distribution of the distance between ground station and reflector. We refer to the distance between ground station and reflector $k$ as the one-dimensional reflector location $r_{k}$. Later we show how the one-dimensional reflector location can be transformed back into the two-dimensional location required in the channel model.

In Fig. 9 we show the estimated distribution of the onedimensional reflector location $p(\hat{r})$. Fig. 9 demonstrates that most reflectors are located within a short distance to the ground station. This result is intuitive as the closer a building is, the more "angular space" it occupies: For example, if a $100 \mathrm{~m}$ wide building is located at a distance of $10 \mathrm{~m}$ to the ground station, it occupies roughly $160^{\circ}$. If the same building is located at a distance of $1 \mathrm{~km}$, it occupies only in $5^{\circ}$.

Nevertheless, as both the results in Fig. 9 show, additional clusters of reflectors exist at larger distances to the ground station. Each cluster is usually caused by a group of buildings or trees. Furthermore, there exist isolated reflectors caused by small isolated single objects, e.g., a metal container on an open field.

We thus propose modeling the one-dimensional reflector location distribution $p(r)$ using three groups of reflectors. Each reflector group is associated with a different distribution:

Close reflectors: The distribution of reflectors in the direct surrounding of the ground station is modeled using a half normal distribution

$$
p_{1}(r) \sim|\mathcal{N}|\left(\zeta_{r, 1, \sigma}^{2}=(213 \mathrm{~m})^{2}\right) .
$$

Cluster reflectors: We model the distribution of reflectors appearing in clusters using a Gaussian mixture model as

$$
p_{2}(r) \sim \frac{1}{\zeta_{r, 2, N}} \sum_{i=1}^{\zeta_{r, 2, N}} \mathcal{N}\left(\zeta_{r, 2, \mu_{i}}, \zeta_{r, 2, \sigma_{i}}^{2}\right) \text {. }
$$

Mean and standard deviation are distributed as $p\left(\zeta_{r, 2, \mu}\right) \sim$ $\mathcal{U}(500 \mathrm{~m}, 2 \mathrm{~km})$ and $p\left(\zeta_{r, 2, \sigma}\right) \sim \mathcal{U}(1 \mathrm{~m}, 50 \mathrm{~m})$. The number of clusters is $\zeta_{r, 2, N}=6$.

Isolated reflectors: An uniform distribution is used to model the distribution of isolated reflectors

$$
p_{3}(r) \sim \mathcal{U}\left(\zeta_{r, 3,1}=0 \mathrm{~km}, \zeta_{r, 3, \mathrm{u}}=4 \mathrm{~km}\right) .
$$

Each distribution is weighted by the factors $\zeta_{r, w 1}=0.65$, $\zeta_{r, w 2}=0.25$, and $\zeta_{r, w 3}=0.1$. These factors can be derived from the distribution shown in Fig. 9. The distribution for the one-dimensional reflector location is

$$
\begin{array}{r}
p(r)=\zeta_{r, w 1} p_{1}(r)+\zeta_{r, w 2} p_{2}(r)+\zeta_{r, w 3} p_{3}(r) \\
\text { with } \sum_{i=1}^{3} \zeta_{r, w i}=1 .
\end{array}
$$

The two-dimensional reflector location $\boldsymbol{r}^{\prime}$ is calculated from the one-dimensional reflector location $r^{\prime}$ (drawn from $p(r)$ ) as follows:

- Close and isolated reflectors, described by $p_{1}(r)$ and $p_{3}(r)$, are uniformly distributed around the ground station on the circle defined by $r^{\prime}$, i.e.,

$$
\boldsymbol{r}^{\prime}=r^{\prime}\left[\cos \varphi^{\prime}, \sin \varphi^{\prime}\right]^{\mathrm{T}} \text { with } \varphi \sim \mathcal{U}(0,2 \pi) .
$$

- The cluster reflectors are distributed as follows: for each cluster we first calculate the cluster mean $\zeta_{r, 2, \mu}^{\prime}$ from $\zeta_{r, 2, \mu}$ as

$$
\boldsymbol{\zeta}_{r, 2, \mu}^{\prime}=\zeta_{r, 2, \mu}^{\prime}\left[\cos \varphi^{\prime}, \sin \varphi^{\prime}\right]^{\mathrm{T}} \text { with } \varphi \sim \mathcal{U}(0,2 \pi) .
$$

The reflectors belonging to each cluster are distributed around the cluster mean $\boldsymbol{\zeta}_{r, 2, \mu}^{\prime}$ by a two-dimensional normal distribution

$$
\begin{array}{r}
\boldsymbol{r}^{\prime}=\boldsymbol{\zeta}_{r, 2, \mu}^{\prime}+\left(r^{\prime}-\zeta_{r, 2, \mu}^{\prime}\right)\left[\cos \varphi^{\prime}, \sin \varphi^{\prime}\right]^{\mathrm{T}} \\
\text { with } \varphi \sim \mathcal{U}(0,2 \pi) .
\end{array}
$$

5) Complex Weight: We model the complex weight $\alpha_{k}(\epsilon)$ relative to FSPL to be a function of the angle $\epsilon$ : the angle $\epsilon$ is calculated as the Euclidean distance between the elevation and azimuth the aircraft is currently seen under and the cones opening direction

$$
\epsilon=\sqrt{\left(\phi_{\mathrm{d}, k}-\phi_{k}\right)^{2}+\left(\theta_{\mathrm{d}, k}-\theta_{k}\right)^{2}} .
$$

Thus, the complex weight is rotationally symmetrical around the center of the cone.

The complex weight $\alpha_{k}(\epsilon, r)$ is calculated as the product of the three processes depicted in Fig. 10

$$
\alpha_{k}(\epsilon)=\alpha_{\mathrm{A}, k} \alpha_{\mathrm{V}, k}(\epsilon) \alpha_{\mathrm{W}}(\epsilon \mid \omega) .
$$

- The average amplitude term $\alpha_{\mathrm{A}, k}$ defines the mean amplitude of the MPC associated with reflector $k$.

- The variable complex weight term $\alpha_{\mathrm{V}, k}(\epsilon)$ characterizes the complex weight of the MPC as a function of the angle $\epsilon$.

- The window term $\alpha_{\mathrm{W}}(\epsilon, \omega)$ defines the drop of the MPC amplitude at the edges of the cone of width $\omega$. 


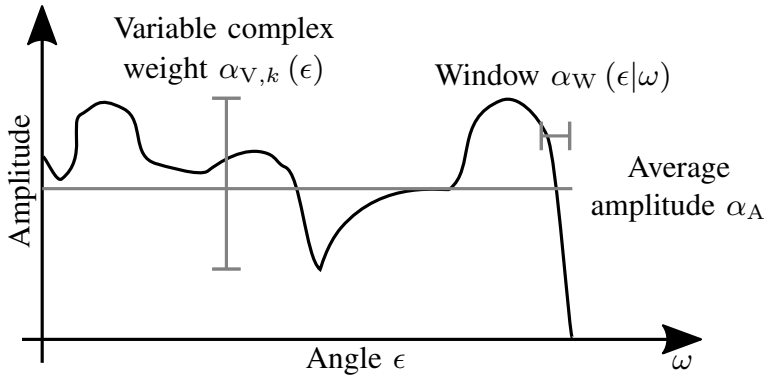

Fig. 10. Visualization of the three processes used for modeling the complex weight of a reflector.

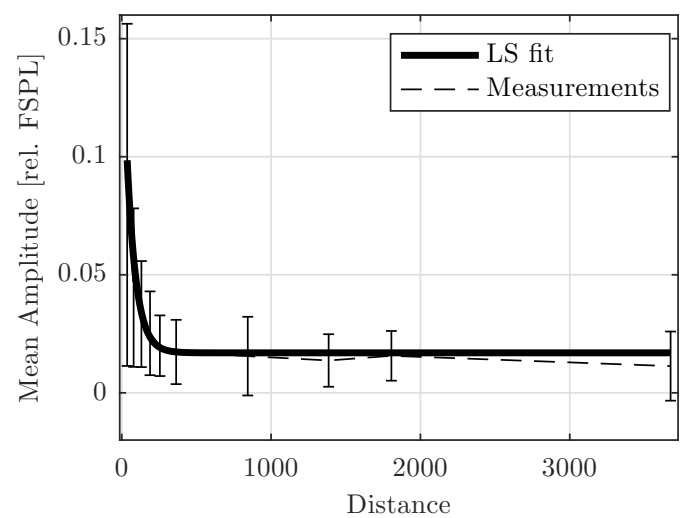

Fig. 11. Mean of average reflector amplitude $\overline{\hat{\alpha}}_{\mathrm{A}}$ as a function of the distance $r$.

Average Amplitude We propose modeling the distribution $p\left(\alpha_{\mathrm{A}} \mid r\right)$ of the average MPC amplitude as the product of two terms

$$
p\left(\alpha_{\mathrm{A}} \mid r\right) \sim p\left(\alpha_{\mathrm{An}}\right) \bar{\alpha}_{\mathrm{A}}(r) .
$$

- The distance dependent mean of the average MPC amplitude is described by $\bar{\alpha}_{\mathrm{A}}(r)$. Thus, $\bar{\alpha}_{\mathrm{A}}(r)$ describes how the mean amplitude of a large number of reflectors at distance $r^{\prime}$ differs from the mean amplitude of a large number of reflectors at distance $r^{\prime \prime}$.

- The distribution around the distance dependent mean is characterized by the distribution $p\left(\alpha_{\mathrm{An}}\right)$., Thus, $p\left(\alpha_{\mathrm{An}}\right)$ describes how the amplitude of a large number of reflectors all located at a fixed distance $r^{\prime}$ varies.

To investigate the distance dependent mean of the average amplitude $\bar{\alpha}_{\mathrm{A}}(r)$ we calculate the average MPC amplitude $\hat{\alpha}_{\mathrm{A}, k}$ associated with each reflector as

$$
\hat{\alpha}_{\mathrm{A}, k}=\frac{1}{M_{k}} \sum_{m=1}^{M_{k}}\left|\hat{\boldsymbol{\alpha}}_{k, m}\right|
$$

Fig. 11 shows the mean and standard deviation of the average amplitude $\hat{\alpha}_{\mathrm{A}}$ against the distance between reflector and ground station $r$. We observe a strong dependence of both mean and standard deviation of the average MPC amplitude on the distance between ground station and reflector: for an increasing distance between ground station and reflector, the standard deviation of the mean decreases.

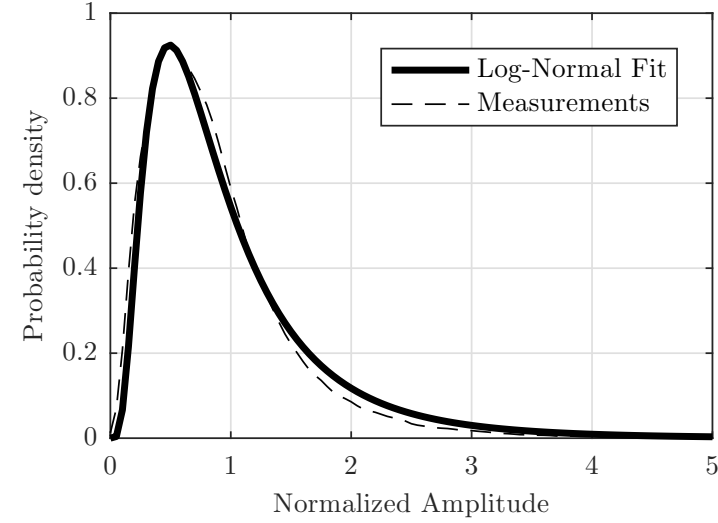

Fig. 12. Distribution of the normalized average reflector amplitude $p\left(\hat{\alpha}_{\mathrm{An}}\right)$.

We model the mean of the average MPC amplitude $\bar{\alpha}_{\mathrm{A}}(r)$ using an exponential function

$$
\bar{\alpha}_{\mathrm{A}}(r)=c_{\alpha_{\mathrm{A}}, 1}+\zeta_{\bar{\alpha}_{\mathrm{A}}, 2} \exp \left\{-\frac{r}{\zeta_{\bar{\alpha}_{\mathrm{A}}, 3}}\right\} .
$$

The coefficients in (27) are least squares (LS) estimated based on the measured data as $\zeta_{\bar{\alpha}_{\mathrm{A}}, 1}=0.017, \zeta_{\bar{\alpha}_{\mathrm{A}}, 2}=0.148$, and $\zeta_{\bar{\alpha}_{\mathrm{A}}, 3}=61.1 \mathrm{~m}$. The result of the fitted function is shown Fig. 11.

As illustrated by the error bars in Fig. 11, for a given distance between ground station and reflector $r^{\prime}$, the average MPC amplitude varies around the distance dependent mean $\bar{\alpha}_{\mathrm{A}}\left(r^{\prime}\right)$. We are now concerned with that variation of the average MPC amplitude around the mean $\bar{\alpha}_{\mathrm{A}}(r)$. To characterize the distribution of that variation we normalize the average MPC amplitudes by the mean $\bar{\alpha}_{\mathrm{A}}(r)$

$$
\hat{\alpha}_{\mathrm{An}, k}=\frac{\hat{\alpha}_{\mathrm{A}, k}}{\bar{\alpha}_{\mathrm{A}}\left(r_{k}\right)} .
$$

Fig. 12 shows distribution of the normalized average MPC amplitude $p\left(\hat{\alpha}_{\mathrm{An}}\right)$. We model the distribution of the normalized average amplitude $p\left(\alpha_{\mathrm{An}}\right)$ using a log-normal distribution

$$
p\left(\alpha_{\mathrm{An}}\right) \sim \log \mathcal{N}\left(\zeta_{\alpha_{\mathrm{An}}, \mu}=-0.23, \zeta_{\alpha_{\mathrm{An}}, \sigma}=0.69\right) .
$$

Therefore, the distribution of the average MPC amplitude $p\left(\alpha_{\mathrm{A}} \mid r\right)$ is given as

$$
p\left(\alpha_{\mathrm{A}} \mid r\right) \sim \log \mathcal{N}\left(\zeta_{\alpha_{\mathrm{An}}, \mu}, \zeta_{\alpha_{\mathrm{An}}, \sigma}\right) \bar{\alpha}_{\mathrm{A}}(r) .
$$

In the following we investigate the complex weight variation of the MPC associated with a reflector. Modeling the variation as a function of the angle $\epsilon$ accounts for the physical observation that, apart from thermal noise, the channel does not change if the aircraft is not moving, e.g., as possible for an airship or a helicopter.

For the analysis of the MPC's complex weight variation we normalize the complex weight of each MPC by its average amplitude

$$
\hat{\boldsymbol{\alpha}}_{\mathrm{V}, k}=\frac{\hat{\boldsymbol{\alpha}}_{k}}{\hat{\alpha}_{\mathrm{A}, k}} .
$$

In Fig. 13 we show the amplitude and phase of an MPC's complex weight $\hat{\boldsymbol{\alpha}}_{\mathrm{V}, k}(\epsilon)$. We observe a correlation of amplitude and phase over the angle $\epsilon$. 


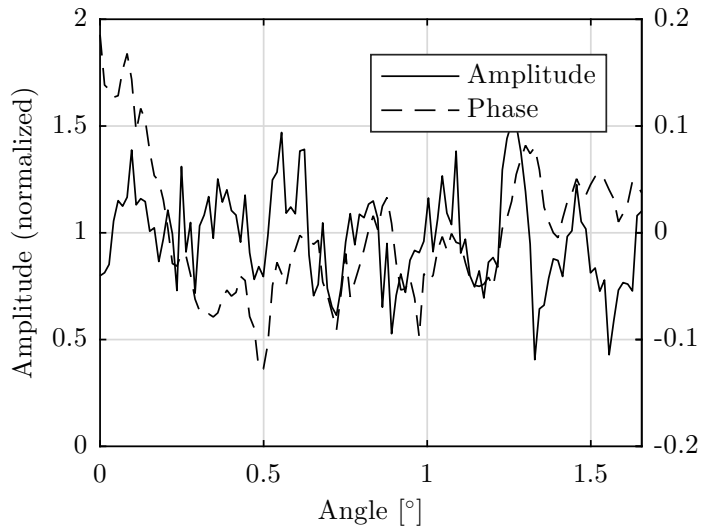

Fig. 13. Distribution of the amplitude, normalized by the mean amplitude of the reflector.

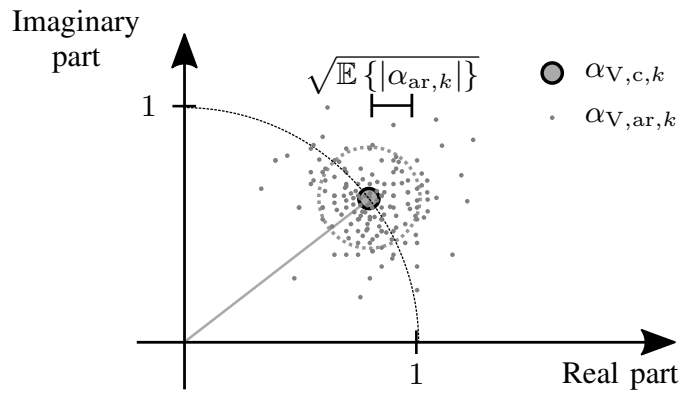

Fig. 14. Proposed modeling approach for the variation of the complex weight.

As illustrated in Fig. 14 we propose modeling the MPC's complex weight $\alpha_{\mathrm{V}}(\epsilon)$ as the sum of two random variables [15]

$$
\alpha_{\mathrm{V}, k}(\epsilon)=\alpha_{\mathrm{V}, \mathrm{c}, k}+\alpha_{\mathrm{V}, \mathrm{ar}, k}(\epsilon) .
$$

The variable $\alpha_{\mathrm{V}, \mathrm{c}, k}$ describes the mean of $\alpha_{\mathrm{V}, k}(\epsilon)$ and $\alpha_{\mathrm{V}, \text { ar }}(\epsilon)$ the variation over the angle $\epsilon$. The amplitude of $\alpha_{\mathrm{V}, \mathrm{c}}$ is ' 1 ' and its phase is uniformly distributed

$$
\alpha_{\mathrm{V}, \mathrm{c}}=e^{\mathrm{jarg}\left(\alpha_{\mathrm{V}, \mathrm{c}}\right)} \quad \text { with } \quad \arg \left(\alpha_{\mathrm{V}, \mathrm{c}}\right) \sim \mathcal{U}(0,2 \pi) .
$$

The random variable $\alpha_{\mathrm{V}, \text { ar }}(\epsilon)$ is drawn from an autoregressive (AR) process.

For the modeling of $\alpha_{\mathrm{V}, k}(\epsilon)$ we require the following information from the measured data:

- The $K$-factor, i.e., the inverse power $\alpha_{\mathrm{V}, a r, k}^{-2}$ of the AR process $^{2}$.

- The model order and pole(s) of the AR process describing $\alpha_{\mathrm{V}, \mathrm{ar}, k}$.

The $\boldsymbol{K}$-factor is calculated as the inverse variance of the normalized complex weight $\hat{\boldsymbol{\alpha}}_{\mathrm{V}, k}$, i.e.

$$
\hat{\mathcal{K}}_{\alpha, k}=\frac{1}{\operatorname{var}\left\{\hat{\boldsymbol{\alpha}}_{\mathrm{V}, k}\right\}}=\left\|\hat{\boldsymbol{\alpha}}_{\mathrm{V}, k}-\sum_{m=1}^{M_{k}} \hat{\alpha}_{\mathrm{V}, k, m}\right\|^{-2}
$$

The distribution of the $K$-factor $p\left(\hat{\mathcal{K}}_{\alpha}\right)$ is shown in Fig. 15 . We model the distribution of the $K$-factor $p\left(\hat{\mathcal{K}}_{\alpha}\right)$ using a

\footnotetext{
${ }^{2}$ The $K$-factor is defined as the ratio between the power of $\alpha_{\mathrm{V}, \mathrm{c}, k}$ and the power of the AR process $\alpha_{\mathrm{V}, \text { ar, } k}$. As the power of $\alpha_{\mathrm{V}, \mathrm{c}, k}$ is equal to '1' we only need to consider the inverse power of the AR process $\alpha_{\mathrm{V}, \text { ar, } k}$.
}

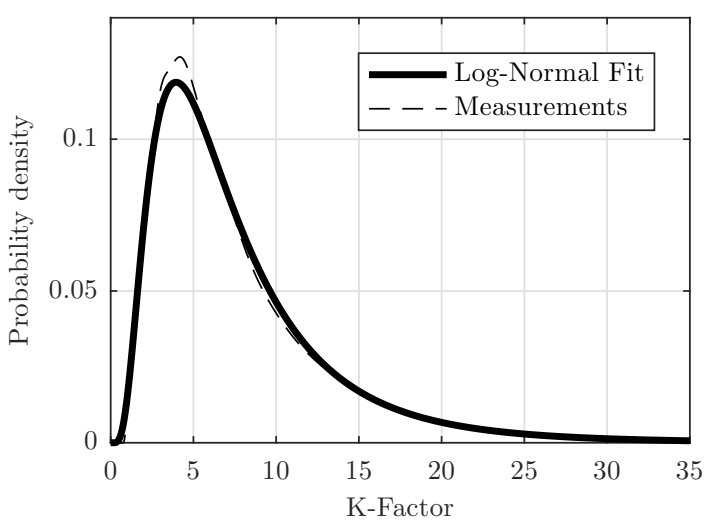

Fig. 15. Distribution of the $K$-factor used to model the complex weight variation.

log-normal distribution

$$
p\left(\hat{\mathcal{K}}_{\alpha}\right)=\log \mathcal{N}\left(\zeta_{\alpha_{\mathrm{V}, K}, \mu}=1.83, \zeta_{\alpha_{\mathrm{V}, K}, \sigma}=0.68\right) .
$$

To select the AR process model order we inspect the distribution of the partial autocorrelation function (PACF) [27]: for every reflector we calculate the PACF and use a Gaussian kernel smoother to estimate the distribution over the reflectors. We observe that for most reflectors the majority of the power is expressed by the first AR coefficient. Therefore, in order to limit the complexity of the channel model, we use an order of ' 1 ' for the AR process.

We calculate variable $\hat{\boldsymbol{\alpha}}_{\mathrm{V}, \mathrm{ar}, k}$ to be modeled by the AR process as

$$
\hat{\boldsymbol{\alpha}}_{\mathrm{V}, \mathrm{ar}, k}=\hat{\boldsymbol{\alpha}}_{\mathrm{V}, k}-\sum_{m=1}^{M_{k}} \hat{\alpha}_{\mathrm{V}, k, m} .
$$

Note however that the vector $\hat{\boldsymbol{\alpha}}_{\mathrm{V}, \mathrm{ar}, k}$ describes measurements of a time series. As we want to model the correlation between two elements in $\boldsymbol{\alpha}_{\mathrm{V}, \mathrm{ar}, k}$ as a function of onedimensional angle $\epsilon$, rather than the time, we resample $\hat{\boldsymbol{\alpha}}_{\mathrm{V}, \mathrm{ar}, k}$ to an angular grid. The resampled vector of complex weights is denoted as $\hat{\boldsymbol{\alpha}}_{\mathrm{V}, \mathrm{ar} \epsilon, k}$. Resampling all complex weights to a grid with the same grid spacing $\epsilon_{\Delta}$ allows the comparison of the AR parameters between the detected MPCs. The pole of the AR process $\hat{\gamma_{\operatorname{ar} k}}$ is estimated based on $\hat{\boldsymbol{\alpha}}_{\mathrm{V}, \operatorname{ar} \epsilon, k}$ using the Vieira-Morf algorithm [28].

Fig. 16 shows the distribution of the absolute value of pole $p\left(\left|\hat{\gamma_{\text {ar }}}\right|\right)$. For the generation of Fig. 16 we use a grid spacing $\epsilon_{\Delta}=0.05^{\circ}$. As the imaginary part of the pole $\operatorname{Im}\left\{\left|\hat{\gamma_{\text {ar }}}\right|\right\}$ is typically negligible we assume the pole to be only real. We model the distribution of $p\left(1-\gamma_{\mathrm{ar}}\right)$ using an Weibull distribution

$$
p\left(1-\gamma_{\mathrm{ar}}\right)=\mathcal{W}\left(\zeta_{\alpha_{\mathrm{V}, \varphi}, A}=0.19, \zeta_{\alpha_{\mathrm{V}, \varphi}, B}=1.34\right) .
$$

Note that the distribution of the pole $p\left(1-\gamma_{\mathrm{ar}}\right)$ has to be truncated at 2 to avoid non-stable AR processes.

The window function takes into account the physical requirement that the MPC amplitudes are time-continuous. In Fig. 17 we show the MPC amplitude distribution at the edges of the cone representing a reflector. We observe a decrease of 


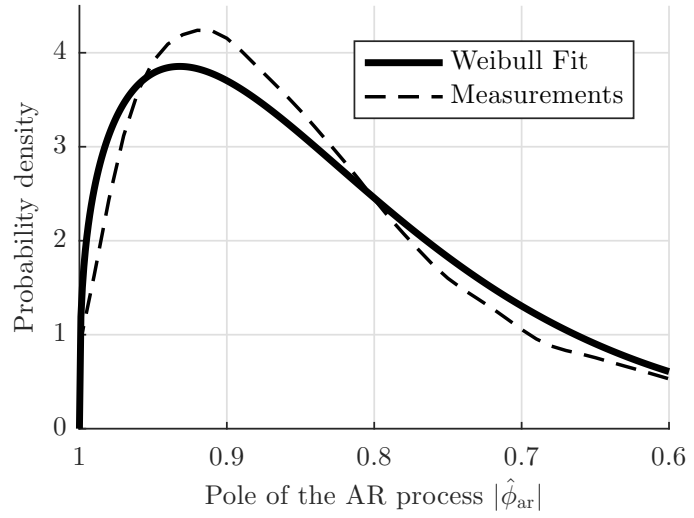

Fig. 16. Distribution of the pole $p\left(\left|\hat{\gamma_{a r}}\right|\right)$ of the AR process used to model the complex weight variation.

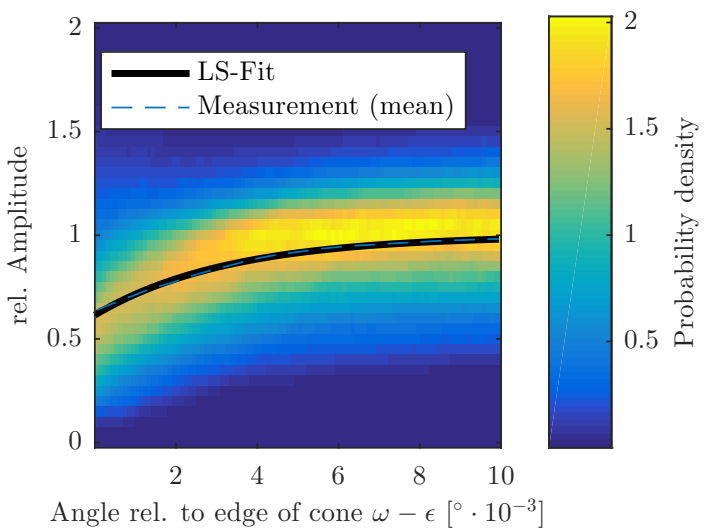

Fig. 17. Distribution of the MPC amplitude close to the edges of the cone representing a reflector.

the amplitude of $\hat{\alpha}_{\mathrm{A}}$ before the signal vanishes. The window function is modeled as

$$
\alpha_{\mathrm{W}}(\epsilon \mid \omega)= \begin{cases}1-e^{-\zeta_{\alpha_{\mathrm{W}}, 1}\left((\omega-\epsilon)-\zeta_{\alpha_{\mathrm{W}}, 2}\right)} & \text { for } \omega_{k}-\epsilon>0, \\ 0 & \text { else. }\end{cases}
$$

The coefficients in (38) are LS estimated based on the measured data as $\zeta_{\alpha_{\mathrm{W}}, 1}=1.7 \times 10^{4}$ and $\zeta_{\alpha_{\mathrm{W}}, 2}=5.5 \times 10^{-5}$.

\section{Antennas}

In this section we investigate the effects of the ground and airborne antennas on the channel [24]. For the inclusion of the antennas we rely on the antenna radiation patterns measured in an anechoic chamber (the antenna is mounted on a ground plane of $30 \mathrm{~cm}$ diameter).

In the following, we analyze how well the antenna radiation patterns are able to predict the measured LoS signal power. To this end, we normalize the measured LoS signal power by the composite gain of both ground and aircraft antenna given the aircraft position and attitude. We term the resulting power as the antenna corrected LoS signal power.

Fig. 18 shows the distribution of the antenna corrected LoS signal power for different (absolute) aircraft roll angles $\left|\varphi_{\mathrm{R}}\right|$.

For small roll angles, i.e., $\left|\varphi_{\mathrm{R}}\right|<5^{\circ}$, the antenna corrected LoS signal power is distributed equally around $0 \mathrm{~dB}$ (relative

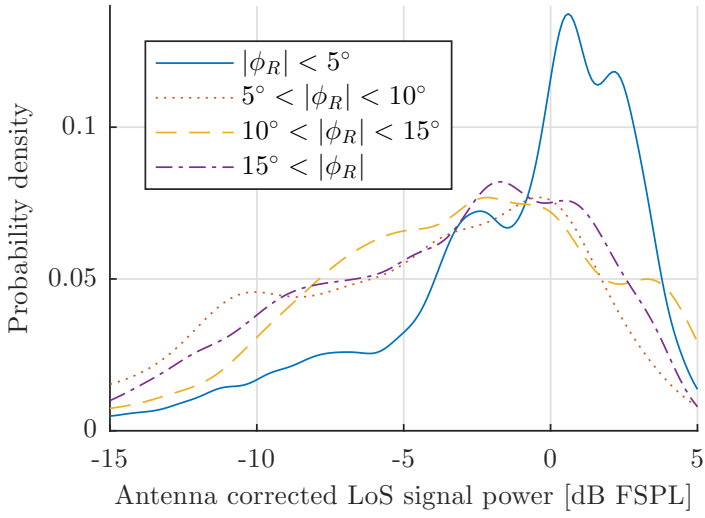

Fig. 18. Influence of the airframe on the LoS signal power as a function of the roll angle. [24]

to FSPL): the mean is $0.1 \mathrm{~dB}$. Therefore, for $\left|\varphi_{\mathrm{R}}\right|<5^{\circ}$ we are able to successfully compensate the gain introduced by the ground and airborne antennas. The wide distribution of the antenna corrected LoS signal power around $0 \mathrm{~dB}$ is attributed to ground multipath propagation. Note that ground multipath propagation on average does not change the mean LoS signal power but only causes a spreading of the LoS signal power distribution.

For increasing roll angles $\left|\varphi_{\mathrm{R}}\right|$ we observe the increasing influence of the airframe. Less power is received than expected as the LoS is shadowed, e.g., by the wings or the tail. We conclude that the proposed modeling approach is very precise for roll angles below $5^{\circ}$.

For larger roll angles an error is introduced. This error could be compensated if the aircraft pattern, i.e., airborne antenna plus airframe, was available: the aircraft pattern could either be measured in an anechoic chamber or estimated based on simulations [29]. However, both techniques require a large amount of resources. Therefore, modeling the influence of the airframe is beyond the scope of this paper: we treat the effects of the airframe as part of the channel.

\section{E. Ground Shadowing}

In this section, we investigate the effect of ground shadowing and we analyze Fig. 19: the figure shows the LoS signal power versus the aircraft position as seen from the ground station. If ground shadowing occurs during the flight the effects of ground shadowing would visible in Fig. 19: first, by a drop of the LoS signal power and second by a "disappearing" of the aircraft, e.g., behind a building, a hill, or the horizon.

We observe that due to the antenna being located on a high building, ground shadowing generally does not arise for the investigated flight trajectories: ground shadowing would only be observed for an extremely low flying aircraft. The channel model parameterization presented in this paper is aimed at normal aircraft flying typical trajectories, i.e., it does not cover aircraft on the ground. Thus, we do not consider ground shadowing by buildings in the following. Note however that if the antenna height was lower ground shadowing would become relevant. 

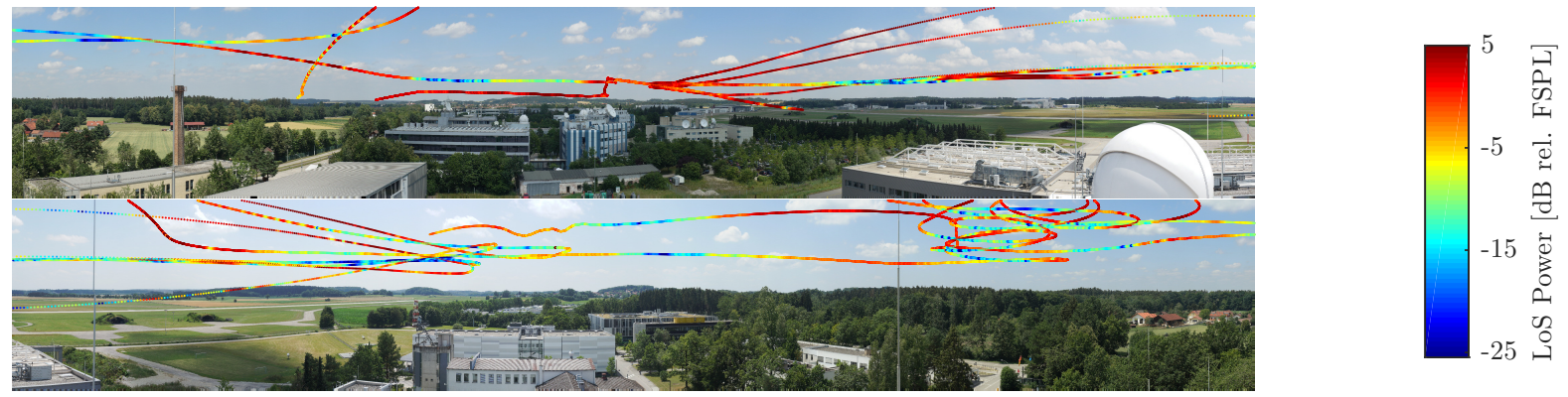

Fig. 19. Color coded trajectories of the aircraft (as seen from the ground station): red color indicates strong LoS signal power, blue color holds for weak LoS signal power.

The only instance where the signal is shadowed is when the aircraft disappears behind the radio horizon (marked with arrows in Fig. 19). However, the channel model parameterization proposed in this paper does not consider the aircraft flying behind the radio horizon.

\section{F. Diffuse Multipath Components}

The diffuse MPCs represent both clusters of reflectors and the effect of scattering. Due to the limited resolution of the MPC parameter estimation objects causing these diffuse MPCs can generally not be located. Diffuse MPCs manifest themselves as a colored noise component in the measured signal. Note however that the influence of the diffuse MPCs on an application system is very small as its typical power is usually below $-20 \mathrm{~dB}$ (relative to FSPL). Thus, for most applications the diffuse MPCs may be omitted to save simulation time.

In order to model the diffuse MPCs we propose a geometrybased stochastic approach: we describe the decay of the power over the delay, i.e., the power delay profile (PDP), by an exponential function [30]. The spread of the power over the Doppler frequency is a function of aircraft position and speed vector.

1) Modeling of the PDP: We model the decay of the diffuse MPCs' PDP (relative to FSPL) using an exponential function

$$
\sigma_{\mathrm{CN}}^{2}(\tau)=\zeta_{\mathrm{CN}, \mathrm{y}} e^{-\tau \zeta_{\mathrm{CN}, \mathrm{s}}} .
$$

The exponential function is defined by the $y$-intersection point $\zeta_{\mathrm{CN}, \mathrm{y}}$ and slope $\zeta_{\mathrm{CN}, \mathrm{s}}$. We estimate the two parameters describing the diffuse MPCs for every channel sounding block based on the residual signal, i.e., the signal minus the detected MPCs. An analysis of the measured data shows that the $y$ intersection point $\zeta_{\mathrm{CN}, \mathrm{y}}$ can be modeled as an exponential function of the link distance $d$

$$
\zeta_{\mathrm{CN}, \mathrm{y}}(d)=e^{\zeta_{\mathrm{CN}, \mathrm{y}, 1}+d \zeta_{\mathrm{CN}, \mathrm{y}, 2}}
$$

with $\zeta_{\mathrm{CN}, \mathrm{y}, 1}=-6.21$ and $\zeta_{\mathrm{CN}, \mathrm{y}, 2}=-0.06 \mathrm{~km}^{-1}$. The slope is modeled constant as $\zeta_{\mathrm{CN}, \mathrm{s}}=1.07 / \mu \mathrm{s}$.

2) Modeling of the Scattering Function: To model the spread of the diffuse MPCs power over the Doppler frequency we make the following assumption: the colored noise is caused by a cloud of $\zeta_{\mathrm{CN}, \mathrm{N}}=200$ scatterers located around the ground station. All scatterers are located in the ground plane.
Their east and north coordinates follow a normal distribution, i.e.,

$$
p\left(\left[d_{\mathrm{CN}, \mathrm{E}}, d_{\mathrm{CN}, \mathrm{N}}\right]^{\mathrm{T}}\right) \sim \mathcal{N}\left(0, \underline{\mathbb{I}}_{2} \zeta_{\mathrm{CN}, \sigma}^{2}\right) .
$$

We adopt the power of each scatterer as described in [31]: given the aircraft position and speed vector we calculate the delay associated which each scatterer. The power at delay $\tau^{\prime}$ bin of the target PDP is equally distributed to all scatterers with a delay falling into the bin $\tau^{\prime}$. Each of the scatterers with the same delay $\tau^{\prime}$ has a different Doppler frequency based on its location: thus, the power at the delay $\tau^{\prime}$ is spread over the Doppler frequency.

\section{VALIDATION}

In the following, we validate the regional airport channel model parameterization. Therefore, we rely on two methods: first, we compare the statistical characteristics of the channel model against results from channel sounding flight trials. Second, we validate the channel model using a application.

\section{A. Quantitative Validation}

The PDP is a key parameter of the channel describing received power over the delay.

In Fig. 20 we show the distribution of the measured PDPs and of the PDPs acquired from the channel model for one realization. The color coded PDP distribution is generated by taking all the flight's 41000 measured or simulated PDPs and estimating the PDFs over every delay bin using a Gaussian kernel smoother [32]. Therefore, each vertical line in Fig. 20 represents a PDF and normalizes to one. The mean power, i.e., the expectation of the PDF, is visualized by the dashed black line. The aircraft in both the measurements and the channel model is flying at altitudes between 3 and $9 \mathrm{~km}$; the link distance is between 20 and $80 \mathrm{~km}$. The delay is taken relative to the LoS signal delay, i.e., we show the excess delay.

The most evident observation is that both measured and modeled PDPs distribution look similar in shape: the overall decay of power over the delay is approximately the same: in both the measured and modeled PDP clusters of MPC are visible. This observation is not only true for the channel model realization leading to Fig. 20(a) but also for other realizations.

When comparing the two PDPs' distributions we observe that the dominant MPCs appear at different delays. However, 


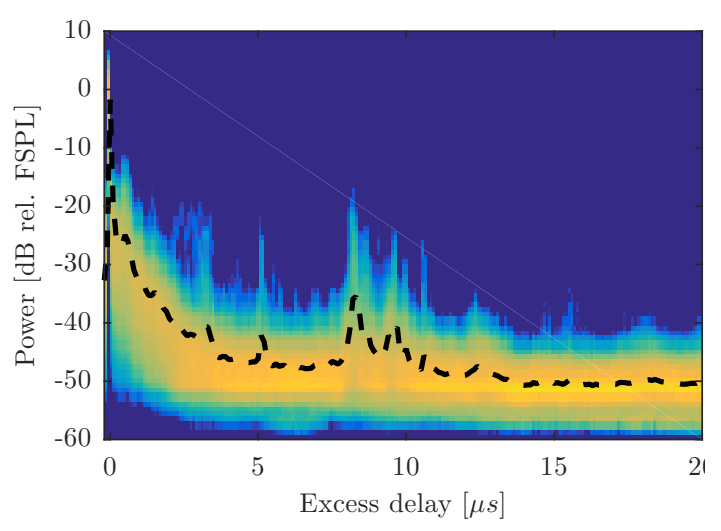

(a) Measurements

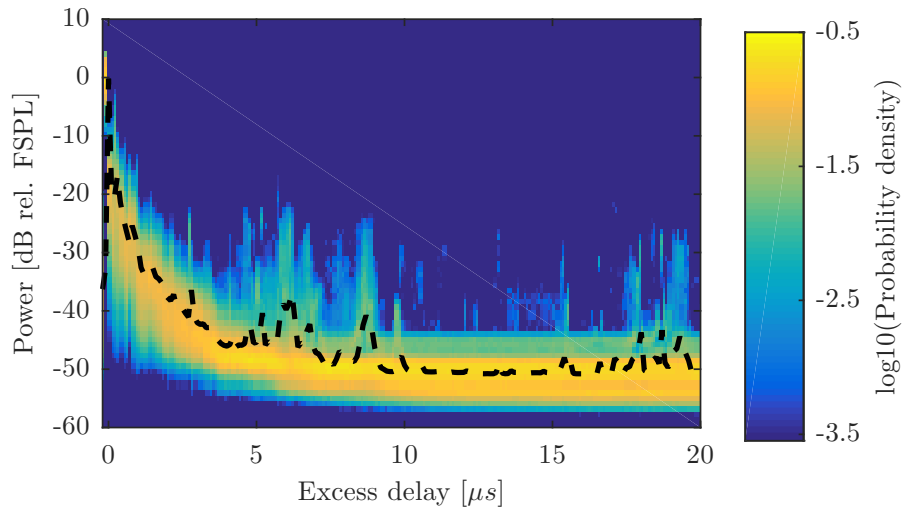

(b) Channel model

Fig. 20. Comparison of the PDPs from measurements and the channel model.

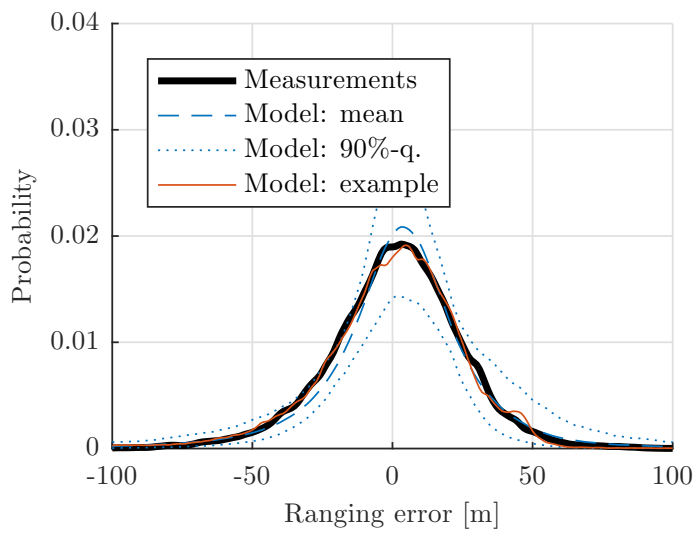

Fig. 21. Distribution of the LDACS ranging error.

this behavior is both expected and desired: as we statistically distribute the reflector locations in each channel model realization the MPCs caused by those reflectors have different delays. Nevertheless, the channel model realizations all exhibit the same characteristics relevant for a terrestrial CNS system.

\section{B. Validation by Application}

The key performance indicator for a channel model is that the channel model can predict the performance of the system it is intended for. Thus, in the following we validate the channel model using LDACS range measurements. We use data from the LDACS flight trials conducted the same year (but during a different flight) as the channel measurements [17].

For the validation by application we rely on two methods: analysis of the statistical error distribution and the ability to reproduce the effects of the channel's non-stationarity on the ranging.

In Fig. 21 we show the PDF of the LDACS ranging error. For the channel model we plot means (dashed blue) and $90 \%$ quantiles (dotted blue) of the distributions based on 50 channel model realizations. We also present an example distribution based on one channel model realization.

From Fig. 21 we observe that the ranging error PDF from measurements and channel model approximately follow a normal distribution: compare the mean ranging error of

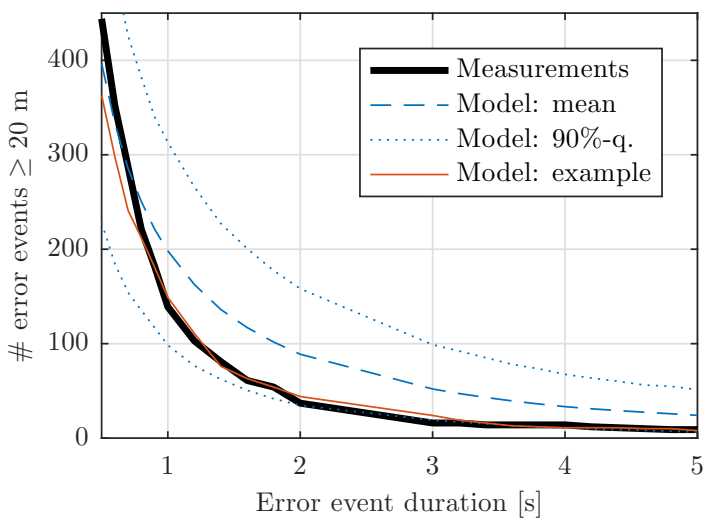

Fig. 22. Occurrence of slowly varying errors exceeding $20 \mathrm{~m}$.

$2.6 \mathrm{~m}$ from measurements with a mean of $1.1 \mathrm{~m}$ over all model realizations. The root-mean-squared error (RMSE) of the measurements is $24 \mathrm{~m}$ and $22 \mathrm{~m}$ for the averaged channel model realizations.

The measured distributions (black) are well within the $90 \%$ quantile of the model (dotted blue); we conclude that the channel model is very well able to replicate the measured ranging error distributions.

The above statistical validation based on the PDF gives no indication how well the effects of the channel's nonstationarity on the ranging performance is represented. Hence, we look at an characteristic of the ranging error of utmost importance in the context of navigation: the existence of slowly varying errors, e.g., an error exceeding $20 \mathrm{~m}$ and existing for a few seconds - an example is visible in Fig. 22. Such slowly varying errors cannot be mitigated by averaging, e.g., using a delay-locked loop (DLL) and cause a severe integrity threat. A stationary statistical channel model is not able to reproduce these kind of errors.

Fig. 22 demonstrates that - unlike previous models - the proposed channel model is able to reproduce slowly varying errors. The figure shows how often such slowly varying errors of a given duration exceeding $20 \mathrm{~m}$ occur during a $1 \mathrm{~h}$ flight. However, we observe from Fig. 22 that the measured curve (black) is on the lower end of the $90 \%$ quantile defined by the 
50 channel model realizations (dotted blue). Such a behavior is not unexpected as the dashed blue curve represents the mean of 50 realizations of the channel model, while the black curve represents only one single flight corresponding to one 'single realization'. The results for individual realizations of the channel model however may be very different due to the large variety of possible geometrical variations for different realizations of the channel model. Nevertheless, within the set of 50 channel model realizations the results for several channel model realizations are similar to the measurement results. One such example is shown (red). Thus, we can conclude that the channel model reproduces slowly varying errors to a similar extent as would be expected in measurements. Although a thorough analysis on how exact the reproduction of slowly varying errors is of great interest, additional flights would be required for such an analysis.

\section{CONCLUSiOn AND OUTLOOK}

Using the GBSCM architecture and measured data this paper presents a parameterization for a regional airport environment: this parameterization is successfully validated against measured data from channel sounding and ranging experiments; we demonstrate a very good match between channel model and measured channel.

With the proposed channel model it is now possible to perform realistic, rapid, and cost effective evaluations of the effect of the channel on the full range of terrestrial CNS systems. Such system evaluations are mandatory for the transition of the CNS infrastructure to new or improved systems.

\section{BIBLIOGRAPHY}

[1] M. Schnell, U. Epple, D. Shutin, and N. Schneckenburger, "LDACS: future aeronautical communications for air-traffic management," IEEE Commun. Mag., vol. 52, no. 5, pp. 104-110, 2014.

[2] L. Eldredge et al., "Alternative positioning, navigation and timing (PNT) study," in International Civil Aviation Organisation Navigation Systems Panel (NSP), Montreal, Canada, 2010.

[3] D. W. Matolak, "Air-ground channels and models: comprehensive review and considerations for unmanned aircraft systems," in Proc. IEEE Aerospace Conference, Big Sky, MT, 2012, pp. 1-17.

[4] M. Rice, A. Davis, and C. Bettweiser, "Wideband channel model for aeronautical telemetry," IEEE Trans. Aerosp. Electron. Syst., vol. 40, no. 1, pp. 57-69, 2004.

[5] D. W. Matolak and R. Sun, "Air-ground channel characterization for unmanned aircraft systems - part I: methods, measurements, and models for over-water settings," IEEE Trans. Veh. Technol., vol. 66, no. 1, pp. 26-44, 2016.

[6] T. J. Willink, C. C. Squires, G. W. Colman, and M. Muccio, "Measurement and characterization of lowaltitude air-to-ground MIMO channels," IEEE Trans. Veh. Technol., vol. 65, no. 4, pp. 2637-2648, 2016.

[7] P. Closas, C. Fernández-Prades, and J. A. FernándezRubio, "A Bayesian Approach to Multipath Mitigation in GNSS Receivers," vol. 3, no. 4, pp. 695-706, 2009.
[8] B. Krach, P. Robertson, and R. Weigel, "An Efficient Two-Fold Marginalized Bayesian Filter for Multipath Estimation in Satellite Navigation Receivers," EURASIP J. Adv. Sig. Proc., vol. 2010, 2010.

[9] A. Molisch, A. Kuchar, J. Laurila, K. Hugl, and R. Schmalenberger, "Geometry-based directional model for mobile radio channels - principles and implementation," European Transactions on Telecommunications, vol. 14, no. 4, pp. 351-359, 2003.

[10] P. Almers et al., "Survey of Channel and Radio Propagation Models for Wireless MIMO Systems," EURASIP J. on Wireless Communications and Networking, no. 1, pp. 1-19, 2007.

[11] A. Molisch and F. Tufvesson, "Propagation Channel Models for Next-Generation Wireless Communications Systems," IEICE Trans. Commun., vol. E-97-B, no. 10, pp. 2022-2034, 2014.

[12] W. Wang, T. Jost, U.-C. Fiebig, and W. Koch, “A Time Variant Outdoor-to-Indoor Channel Model for Mobile Radio Based Navigation Applications," vol. 2015, 2015.

[13] H. Liu, H. Darabi, P. Banerjee, and J. Liu, "Survey of Wireless Indoor Positioning Techniques and Systems," vol. 37, no. 6, pp. 1067-1080, 2007.

[14] T. Santos, F. Tufvesson, and A. Molisch, "Modeling the ultra-wideband outdoor channel: model specification and validation," IEEE Trans. Wireless Commun., vol. 9, no. 6, pp. 1987-1997, 2010.

[15] T. Jost, W. Wang, U.-C. Fiebig, and F. Pérez-Fontán, “A wideband satellite-to-indoor channel model for navigation applications," IEEE Trans. Antennas Propag., vol. 62, no. 10, pp. 5307-5320, 2014.

[16] N. Schneckenburger, T. Jost, D. Shutin, M. Walter, T. Thiasiriphet, M. Schnell, and U.-C. Fiebig, "Measurement of the L-band air-to-ground channel for positioning applications," IEEE Trans. Aerosp. Electron. Syst., vol. 52, no. 5, pp. 2281-2297, 2016.

[17] T. Thiasiriphet, N. Schneckenburger, and M. Schnell, "Ranging with LDACS: results from measurement campaign," in Proc. ICNS, Herndon, VA, 2016.

[18] N. Schneckenburger et al., "Modeling the air-ground multipath channel," in Proc. EuCAP, Paris, France, 2017.

[19] J. D. Parsons, The Mobile Radio Propagation Channel, 2nd. West Sussex, United Kingdom: Wiley, 2000.

[20] G. H. Millman, "Atmospheric effects on VHF and UHF propagation," in IEEERE International Convention, vol. 46, 1958, pp. 1492-1501.

[21] ITU-R, "P.1008-1: Reflection from the surface of the Earth,” Tech. Rep., 1990.

[22] — "P.527-3: Electrical characteristics of the surface of the earth," Tech. Rep., 1992.

[23] D. W. Matolak and R. Sun, "Air-ground channel characterization for unmanned aircraft systems - part III," IEEE Trans. Veh. Technol., vol. 66, no. 8, pp. 66076618, 2017.

[24] N. Schneckenburger, D. Matolak, T. Jost, U.-C. Fiebig, and G. Del Galdo, "A geometrical-statistical model for 
the air-ground channel," in Proc. DASC, St. Petersburg, FL, 2017.

[25] N. Schneckenburger, T. Jost, D. Shutin, M. Walter, U.-C. Fiebig, and G. Del Galdo, "Reflector localization for geometrical modeling the air-ground channel," IEEE Trans. Veh. Technol., vol. 67, no. 9, pp. 7994-8008, 2018.

[26] M. Ait Ighil, "Enhanced physical-statistical simulator of the land mobile satellite channel for multipath modelling applied to satellite navigation systems," $\mathrm{PhD}$ thesis, Université de Toulouse, 2013.

[27] G. Box, G. Jenkins, and G. C. Reinsel, Time Series Analysis: Forecasting and Control. Wiley, 2008.

[28] S. L. Marple, Digital Spectral Analysis: With Applications. Prentice Hall, 1987.

[29] K. J. Keeping and J. C. Sureau, "Scale model pattern measurements of aircraft 1-band beacon antennas," Lincoln Laboratory, Tech. Rep., 1975.

[30] A. Richter, "Estimation of radio channel parameters: Models and algorithms," PhD Thesis, TU Ilmenau, Ilmenau, Germany, 2005.

[31] T. Jost, W. Wang, and M. Walter, "A geometry-based channel model to simulate an averaged power delay profile," IEEE Trans. Antennas Propag., no. 99, 2017.

[32] C. M. Bishop, Pattern recognition and machine learning. Secaucus, NJ: Springer, 2006. 